\title{
Experimental haemarthrosis produces mild inflammation associated with intracellular Maltese crosses*
}

\author{
SUNG-JAE CHOI, H RALPH SCHUMACHER JR, AND \\ GILDA CLAYBURNE
}

From the University of Pennsylvania, School of Medicine and Veterans Administration Medical Center, Philadelphia, PA

SUMMARY Injection of autologous blood into rabbit joints induces an inflammatory reaction with Maltese cross-like birefringent spherulites. Similar microspherules seen in human joint fluids may be formed by lipids derived from breakdown of erythrocytes and other cells.

Key words: polarised light, synovial fluid, lipid liquid crystals, crystals, electron microscopy, rabbits, arthritis.

Intracellular birefringent liquid microspherules appearing as Maltese crosses have been reported in synovial fluids (SF) of patients with unexplained acute or chronic arthritis. ${ }^{1-4}$ These appeared as multilayered membranous arrays by electron microscopy. ${ }^{3}$ The origin of the spherulites in such patients was not explained. Since mild inflammation with ultrastructually similar inclusions has been reported to occur after injections of blood ${ }^{5}$ we proposed that birefringent microspherules might be seen with polarised light. This could suggest membrane lipids of lysed cells as at least one source for the unexplained spherulites.

We have therefore studied experimentally produced haemarthrosis in the rabbit and performed the first sequential light microscopic examinations of their SF.

\section{Subjects and methods}

\section{R A B B I T S}

Five mature male albino New Zealand rabbits weighing $3 \cdot 0-3.5 \mathrm{~kg}$ were sedated with ketamine 40 $\mathrm{mg} / \mathrm{kg}$ and zylazine $5 \mathrm{mg} / \mathrm{kg}$ intramuscularly. In each rabbit $0.5 \mathrm{ml}$ of arterial blood was obtained from the animal's own ear and under aseptic conditions

Accepted for publication 22 May 1986.

Correspondence to Dr H Ralph Schumacher. Arthritis-Immunology Center, Veterans Administration Medical Center, University and Woodland Avenues, Philadelphia, PA 19104, USA.

${ }^{*}$ Presented in part at the 49th Annual Meeting of the American Rheumatism Association. New Orleans, LA, June 1986. immediately injected without any additives into the right knee. The left knee served as an uninjected control. At two, four, and six days after single injections of blood and one week and two weeks after twice weekly injections animals were autopsied and SF and synovial membranes (SMs) were examined by polarised light, regular light, and electron microscopy.

SYNOVIAL FLUIDS AND MEMBRANES

The SF when present was analysed manually for leucocyte count (with $0.3 \%$ saline as a diluent). A wet preparation was immediately examined under polarised light and Wright's stained smears were done for differential leucocyte counts. For lipid stain two or three drops of SF were placed on a slide, mixed with an equal amount of Sudan black B, and the preparation was examined immediately. ${ }^{6}$ The remaining fluid was prepared for transmission electron microscopic study by centrifuging immediately, fixing pellets in half strength Karnovsky's paraformaldehyde-glutaraldehyde fixative, and processing as previously described. ${ }^{7-9}$

Synovial membrane specimens for light microscopy were fixed in buffered formalin and processed through routine paraffin embedding, sectioned, and examined after staining with haematoxylin and eosin.

\section{Results}

SFs of blood injected knees showed leucocyte 


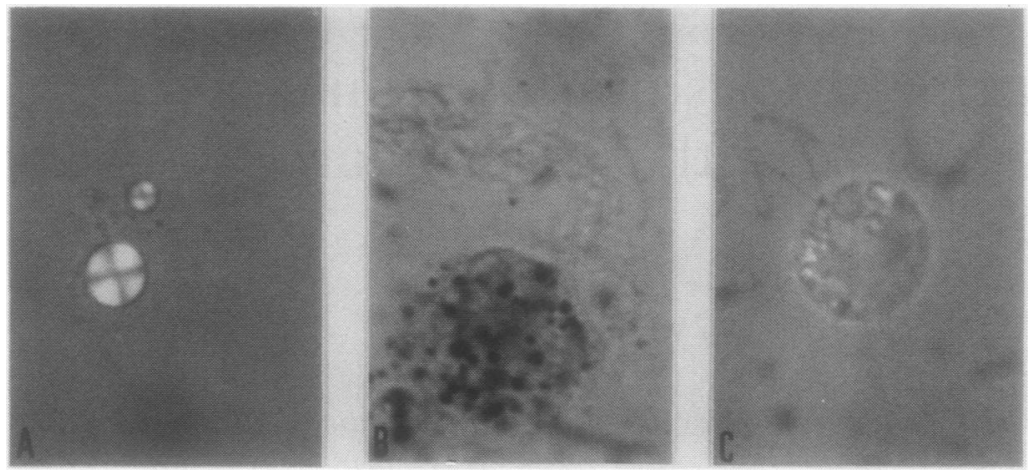

Fig. 1 (A) Maltese cross-like inclusions in a degenerating synovial fluid leucocyte. Wet preparation. Compensated polarised light. (B) Sudan black B positive inclusions in joint fluid leucocyte. Regular light. (C) Other irregular birefringent material in an intact leucocyte. Wet preparation. Compensated polarised light.

counts of $1600-7100 / \mathrm{mm}^{3}\left(1 \cdot 6-7 \cdot 1 \times 10^{9} / 1\right)$, while uninjected control knees had 500-800 leucocytes/ $\mathrm{mm}^{3}\left(0 \cdot 5-0 \cdot 8 \times 10^{9} / \mathrm{l}\right)$ (Table 1). Examination of wet smears of SFs from all blood injected joints by compensated polarised light showed intracellular positively birefringent spherulites appearing as Maltese crosses (Fig. 1A) and staining with Sudan black B (Fig. 1B). Other birefringent inclusions were irregular and less clearly Maltese crosses, but probably similar in origin (Fig. 1C). Many of these appeared as Maltese crosses with very careful focusing. Control joints had no birefringent material. Approximately $10-30 \%$ of leucocytes from blood injected joints contained Maltese cross-like spherulites. SFs of joints repeatedly injected with blood had more abundant spherulites than those of once injected joints.

Histology of SMs of blood injected joints showed mild to moderate proliferation of synovial lining cells with infiltration of polymorphonuclear leucocyte (PMN), lymphocyte, and mononuclear cells. At two days PMN infiltration was prominent throughout superficial synovium and at four and six days there were many mononuclear cells and lymphocytes with only a few PMN.

Electron micrographs of SFs and SMs of bloo injected joints showed osmiophilic multilayered membranous arrays in PMN, macrophages, and

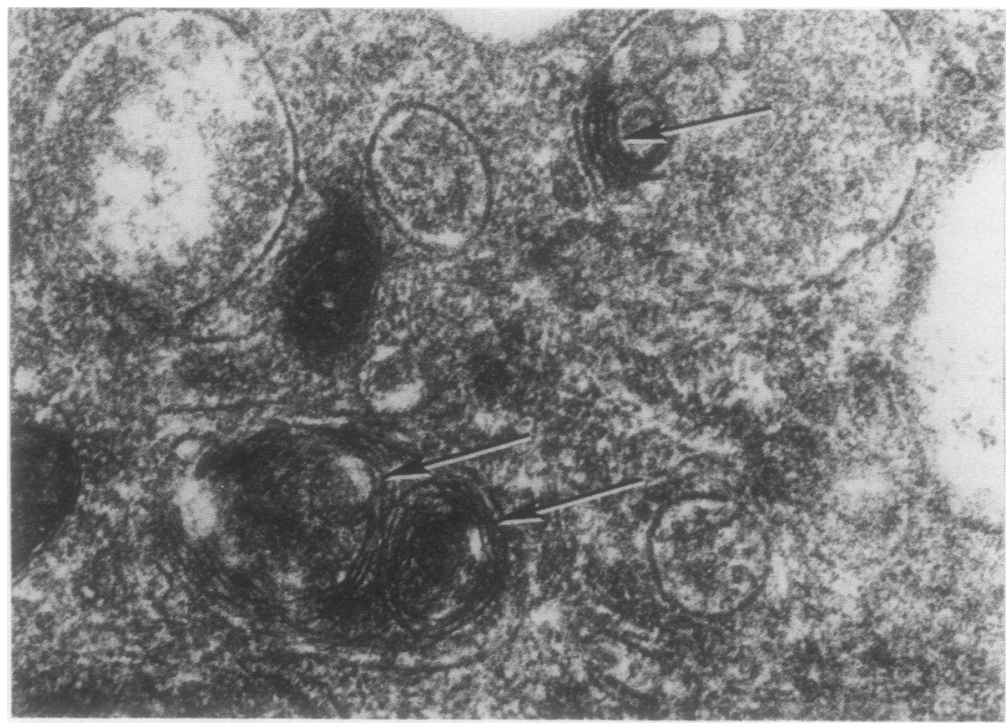

Fig. 2 Electron micrograph showing membranous whorls in vacuoles of a synovial lining cell after two injections of blood into the joint (rabbit 16). 


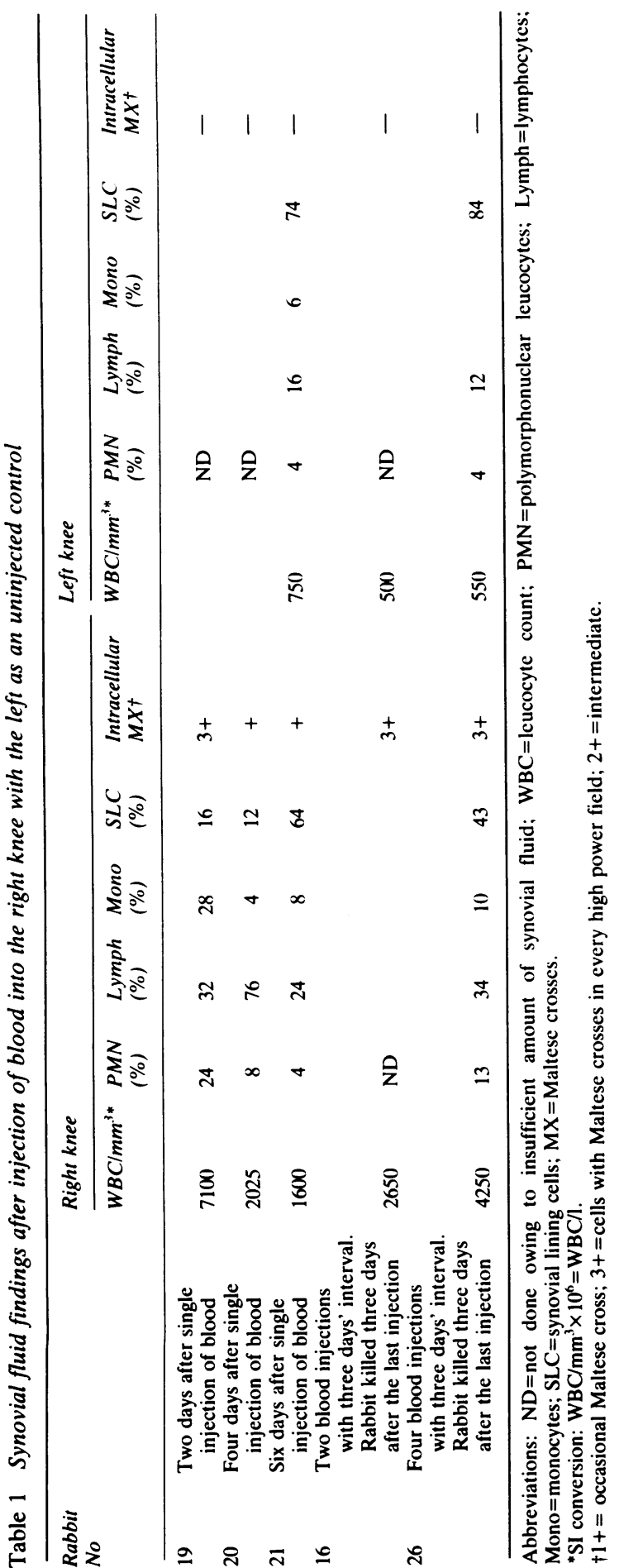

synovial lining cells (Fig. 2). No similar inclusions were seen in the control joints.

\section{Discussion}

Different types of synovial lipid bodies have been reported in a variety of disorders. ${ }^{1-3}$ 10-16 Cholesterol crystals have been seen in rheumatoid, osteoarthritic, and other chronic effusions. ${ }^{12-16} \mathrm{Ex}$ tracellular and intracellular non-birefringent lipid globules have been reported in traumatic arthritis $^{1011}$ and rarely in aseptic necrosis ${ }^{17}$ and rheumatoid arthritis. ${ }^{14}$

Birefringent lipid microspherules appearing as Maltese crosses have occasionally been observed without comment during synovial fluid analysis. ${ }^{18}$ Recently such microspherules have been reported in the synovial fluid of one patient with otherwise unexplained acute monoarthritis, ${ }^{1}$ in the synovial fluid and synovial lining cells of a patient with chronic unexplained arthritis, ${ }^{2}$ and in the synovial fluids of three other patients with unexplained acute monoarthritis. ${ }^{3}$ SFs in all these case reports were described as having erythrocyte (red blood cell (RBC)) counts from $1350 / \mathrm{mm}^{3}\left(1.35 \times 10^{9} / \mathrm{l}\right)$ to grossly bloody fluid. These findings suggested to us that $\mathrm{RBC}$ should be considered as one likely source of Maltese cross-like spherulites. Our SFs of blood injected rabbit joints showed birefringent spherulites similar to those of human cases, ${ }^{1-3}$ thus supporting this possibility.

Electron microscopic findings of SFs and SMs in the blood injected rabbit joints showed whorled membranous arrays similar to those myelin-like figures described in SF in one human case report ${ }^{3}$ and in liposome injected rabbit joints. ${ }^{19}$ Roy and Ghadially had previously shown similar myelinoid (membranous) bodies in the synovial cells of rabbit joints after intra-articular injection of autologous blood. ${ }^{5}$ It was suggested that these membranous structures might represent hydrated lipidic residues derived from the cholesterol rich erythrocyte membrane. ${ }^{20}$ Roy and Ghadially, however, only examined SMs, not SFs, in blood injected rabbit joints and had not carried out studies with polarised light.

Our studies and the literature noted thus suggest that erythrocyte membranes can be the source of Maltese cross-like birefringent inclusions in synovial fluid cells. Whether these are an important cause of the inflammation seen after blood injection or human haemarthrosis remains to be determined. Other elements in the blood could also contribute to the inflammatory reaction. Most patients with Maltese crosses described in joint fluids have not recalled joint trauma as the source of the erythro- 
cytes. Some RBC are common in many joint fluids; ${ }^{21}$ RBC may arise from subtle mechanical trauma or can escape from congested vessels in inflammatory arthritis. Leucocyte membranes might also be a source of membrane lipids in effusions without RBC. Small numbers of Maltese cross-like spherulites certainly may occur secondarily and be coincidental findings in a variety of known joint diseases. We frequently see rare birefringent spherulites in chronic inflammatory arthritis. Large numbers of such spherulites, however, from RBC or other origins may very well contribute to the joint inflammation as was seen in these rabbits.

\section{References}

1 Weinstein J. Synovial fluid leukocytosis associated with intracellular lipid inclusions. Arch Intern Med 1980): 140: 56(-1.

2 Schlesinger P A. Stillman M T. Peterson L. Polyarthritis with birefringent lipid within synovial fluid macrophages: case report and ultrastructural study. Arthritis Rheum 1982: 25: 1365-8.

3 Reginato A J. Schumacher H R. Allan D A. Rabinowitz J L. Acute monoarthritis associated with lipid liquid crystals. Ann Rheum Dis 1985: 44: 537-43.

4 Trostle D C. Schumacher H R. Medsger T A. Kapoor W N. Microspherule associated acute monoarticular arthritis. Arthritis Rheum (in press).

5 Roy S. Ghadially F N. Pathology of experimental hacmarthrosis. Ann Rheum Dis 1966; 25: 402-15.

6 Malinin T I. Pekin T J. Bauer H. Zvaifler N J. Vacuoles in synovial fluid lcukocytes. Am J Clin Pathol 1966; 45: 728-31.

7 Schumacher H R. The microvasculature of the synovial membrane of the monkey: ultrastructural studies. Arthritis Rheum 1969: 12: 387-4(1)4.
8 Schumacher H R. Synovial membrane and fluid morphologic alterations in early rheumatoid arthritis: microvascular injury o and virus-like particle. Ann NY Acad Sci 1975: 256: 39-64.

9 Schumacher H R. Palindromic onset of rheumatoid arthritis: $\overline{\bar{u}}$ clinical. synovial fluid. and biopsy studies. Arthritis Rheum 1982; 25: 361-9.

10) Graham J. Goldman J A. Fat droplets and synovial fluid leukocytosis in traumatic arthritis. Arthritis Rheum 1978: 21: 76-80.

11 Ryan W E. Ellefson R D. Ward L E. Lipid svnovial effusion: unique occurrence in systemic lupus erythematosus. Arthritis Rheum 1973: 16: 759-64.

12 Ettlinger R E. Hunder G C. Synovial effusions containing cholesterol crystals, report of 12 patients and review. Mayo Clin Proc 1979: 54: 366-74.

13 Zuckner J. Uddin J. Ganter G E. Dorner R W. Cholesterol crystals in synovial fluid. Ann Intern Med 1964: 60: 436-46.

14 Newcombe D S. Cohen A S. Chylous synovial effusion in rheumatoid arthritis, clinical and pathogenetic significances. Am J Med 1965: 38: 156-64.

15 Griffin P E, Bole G G. Cholesterol-containing synovial effusions and cholesterosis of the synovial membrane. Univ Mich Med Cent J 1969: 35: 170)6.

16 Fam A G. Pritzker K P H. Cheng P T. Little A H. Cholesterol crystals in ostcoarthritic joint cffusion. $J$ Rheumatol 1981: 8: 273-80.

17 Bravo J F. Herman J D. Smyth C J. Musculoskeletal disorders after renal homotransplantation. Ann Intern Med 1967; 66: 87-104.

18 Wild J H. Zvaifler $\mathrm{N} \mathrm{J}$. An office technique for identifying crystals in synovial fluid. Am Fam Physician 1975: 12: 72-81

19 Choi S J. Schumacher H R. Clayburne G. Rothfuss M Liposome-induced synovitis in rabbits |Abstract|. Arthrit Rheum 1985: 28: S79.

20 Ghadially F N. Hacmorrhage and hemosiderin. J Submicro Cytol 1979: 11: 271-9.

21 Ropes M W. Bauer W. Sinovial fluid changes in joint disease. Cambridge. Mass: Harvard Univ Press, 1953 